\title{
A cognitive prosthesis and communication support for people with dementia
}

\author{
Norman Alm ${ }^{1}$, Maggie Ellis ${ }^{2}$, Arlene Astell ${ }^{2}$, Richard Dye ${ }^{1}$, Gary Gowans ${ }^{3}$ and Jim Campbell ${ }^{3}$ \\ ${ }^{1}$ Division of Applied Computing, University of Dundee, Dundee, DD1 4HN, Scotland \\ ${ }^{2}$ School of Psychology, University of St Andrews, St Andrews, KY16 9JU, Scotland \\ ${ }^{3}$ Department of Computer-Aided Design, Duncan of Jordanstone College of Art and Design \\ University of Dundee, Dundee, DD1 4HT, Scotland
}

$\underline{\text { Running title }}$

Cognitive prosthesis and communication 


\section{THE POTENTIAL OF THE COMPUTER AS A COGNITIVE PROSTHESIS}

The potential of computers as augmenters of human intellect has been noted at least since Engelbart's insights (Engelbart 1963). The term 'cognitive prosthesis' has been used to identify the application of this approach to human-computer partnership to problems caused by impairments in cognitive ability. Kirsh, Levine, Fallon-Krueger, and Jaros (1987) set out a useful description of how a cognitive prosthesis might operate in practice. A cognitive prosthesis should provide a compensatory strategy for people with a cognitive impairment that, when added to the user's environment, increases their ability to function effectively. It has been speculated that advances in technology could eventually allow a cognitive prosthesis system to act as a 'companion' for a person with cognitive impairments, helping them by monitoring their activities and offering appropriate prompts and advice (Vanderheiden 1990). Arnott emphasised the importance of drawing the boundaries between the person and the computer so that the person was ultimately in overall control, even if the computer was performing cognitive tasks on their behalf (Arnott 1990). Alm, Waller and Newell have argued that systems which assist non-speaking people to communicate will need to operate at the level of a cognitive prosthesis if realistic rates of communicate are to be achieved (Alm, Waller, Newell 1996). Cole and his team have developed a range of compensatory systems for people with acquired cognitive impairments, and emphasise in their work the need for highly personalisable systems (Cole 1999).

Computers do seem to have the potential to act as a kind of scaffolding to support cognitive tasks, taking over some functions which have been affected by illness, accident, or aging. Computers might also provide prompts for daily living, if they were able to track successfully the user's sequence of tasks and actions. One area which such developments would be welcome is in supplying support for elderly people with dementia and their carers. We have been working in this idea through a number of exploratory projects. The starting point for some of the thinking behind these projects was our previous work on helping non-speaking people to communicate through computer-based systems.

\section{SUPPORT FOR COMMUNICATION BY PHYSICALLY IMPAIRED NON-SPEAKING PEOPLE}

Some of the work done to improve communication systems for people who are unable to speak due to physical impairment has involved developing innovative ways of providing computer generated support for the communication. This support has at times acted as a cognitive prosthesis, by assisting the communication through taking over some of the cognitive processes involved when we interact through language.

For severely physically impaired non-speaking people, even with current speech output technology, speaking rates of 2-10 words per minute are common, whereas unimpaired speech proceeds at 150-200 words per minute. In an attempt to improve this, a certain amount of progress has been made in the area 
of using computers to replace or augment some of the cognitive aspects of communication. Although the cognitive processes underlying language use are incompletely understood, a number of theories which attempt to explain language use have been used to improve the functionality of communication systems for non-speaking people. This approach to the problem usually involves paying attention to the pragmatics of language, that is, language as it is used in context. Instead of focusing on the word-byword building blocks which compose an utterance, taking a 'bottom up' approach, the interaction as a whole is analysed, paying attention to its goals, taking a 'top-down' approach to the communication. This may well be a realistic simulation of the natural process, since the production of speech by an unimpaired speaker occurs at such a rate that conscious processing and controlling of the speech at a micro-level is not possible. In common with other learned skills, speech is produced to some extent, automatically, with the speaker being aware of giving high level instructions to the speech production system, but leaving the details of its implementation to the system.

A number of projects have been carried out taking this approach to communication, and increasing the communication rate considerably through the use of prestored conversational material. The CHAT prototype gave the user the ability to move easily through the more formulaic stages of daily interactions, such as openings, closings, and giving feedback to other speakers (Alm, Arnott \& Newell, 1992). Another system, called TALK, experimented with modelling the way in which topic shifting occurs in a step-wise fashion during a casual conversation (Alm, Todman, Elder \& Newell, 1993). Work has also been carried out investigating the usefulness of providing users with scripts for use in common daily situations (Dye, Alm, Arnott, Harper \& Morrison, 1998).

What these projects have in common is providing the user with a partial model of communication, a structure within which reusable utterances are stored. Because the structure closely follows the way a conversation proceeds, utterances are made available to the user in a timely and appropriate way. Having developed such a structure in order to make it easier for users to have the right utterance at the right time, it became clear that the same structure could act as a prompt for communication, as well as being a passive store of useful utterances. This aspect of such systems in fact caused some debate about the desirability of prompting conversational directions, when one overall intention was to improve the individual's conversational control. Because the amount of conversational control possible at 2-10 words per minute is severely restricted, this argument seems to be a matter of judgement about tradeoffs. It is relevant to note, however, that opportunism is a feature of a great deal of casual conversation in any case.

An opportunity presented itself to examine a positive impact of the prompting ability of these systems, and also to see a communication system as a type of cognitive prosthesis, with the development of a communication system for people with aphasia, where communicative and cognitive difficulties were inseparable (Waller, Dennis, Cairns, Brodie, Newell \& Morrison, 1995). This system stored personal narratives which the person with aphasia entered with the help of family members. Once entered, the narrative could then be called up by the person and spoken out sentence by sentence, to create interaction sessions which were enjoyed both by the person using the system and their conversation partners. This provision of prompts for communication, based on a model of the interaction, is an idea which has now led to a new area of development : cognitive and communication support for people with dementia. 


\section{THE CHALLENGE OF DEMENTIA}

As the proportion of the elderly population in many countries is increasing sharply, the number of older people who have dementia or other difficulties and are in need of support in their daily life will correspondingly increase. The numbers of people in the UK over the age of 65 are predicted to increase from 9.25 million in 1996 to 12 million in 2021. The number of people aged over 75 will have doubled and the number over 90 will have more than tripled. The USA Census Bureau states that the chances of having a disability increases with age, and shows that more than half of the population who are 65 or over have a disability (U.S. Bureau of the Census, 1995). A significant proportion of these disabilities are cognitive in nature. Currently one in four of those over 80 have dementia. Along with the social and economic challenges this brings, people with dementia also require support for the practicalities of daily living. In addition, the quality of life and well being of the person with dementia and their carers must also be considered.

Dementia, which involves the impairment of short term memory, presents a serious challenge for both the person with dementia and for their family and carers. It can rule out many social activities and interactions, since these depend on a working short term memory for effective participation. Impairment of short-term memory can affect even the basic ability to communicate. As Azuma and Bayles point out: 'Perhaps the most devastating consequence of dementia, to both caregivers and patients, is the progressive decline in communicative ability resulting from deteriorating memory and intellect' (Azuma \& Bayles 1997).

\section{REMINISCENCE AS A COMMUNICATION SUPPORT}

It has been suggested that improving the relationship between carers and people with dementia could be a most appropriate aim of dementia care (Jackson, 1991). It is also recognised that the provision of a positive interaction, at whatever level a person with dementia understands it, can be considered a successful intervention (Woods, 1994). Finding ways to promote communication in people with dementia is vitally important for a number of reasons. First, communication is such a fundamental part of being human that when people are no longer able to communicate successfully they are treated as somehow less than human. This dehumanisation is, sadly, commonly seen in the treatment of people with dementia (Kittwood, 1990). Second, caring for someone with dementia can be frustrating and upsetting. When communication fails, carers are left to infer intention and meaning from behaviour and this can have negative consequences, such as believing incorrectly that someone is deliberately being difficult. Third, there is a progressive and uneven breakdown in communicative abilities in dementia. Thus the apparent loss of some abilities does not mean a person can no longer communicate altogether. Consequently, interventions must be targeted at the relatively intact functions (Astell \& Harley, 1998; Azuma and Bayles, 1997; Rau, 1993).

Short-term memory impairments can of course make various aspects of conversation very difficult and frustrating for the conversation partner. However, activities that do not require the person with dementia to keep a conversation topic active can provide a satisfying and interesting interaction for both parties. It is important to discover ways of continuing to interact with the person with dementia which as far as possible provide carers with a picture of the whole person and not just a set of needs, and which give the person with dementia a chance to experience satisfying communication. One technique that has proved 
effective for both the person with dementia and caregiver in this respect is reminiscence work (Baines, Saxby \& Ehlert, 1987; Finnema, Dröes, Ribb \& Van Tilburg, 1999). This takes advantage of the fact that long-term memory may be relatively intact, even where the person's short-term memory is severely affected. Reminiscence sessions are typically carried out by creating a scrapbook of photos and other memorabilia, and may incorporate audio and video tapes. These materials become not only a memory aid, but a support to communication, partly replacing the person's own lost ability to deal with immediate memories (such as what they said five minutes ago), while encouraging them to employ their still effective long-term memory (such as what happened forty years ago).

Reminiscence is of course a natural and valuable form of interaction for older people in general. It can give them 'a dignity, a sense of purpose, in going back over their lives and passing on valuable information to a younger generation' (Thompson, 1978). Woods has identified several aims for this type of experience: 'Reminiscence [has] ... a variety of goals, including increased communication and socialisation, and providing pleasure and entertainment' (Woods, 1999). As well as being valuable to older people in general, reminiscence can also serve as an important tool to empower older people who have dementia (Feil, 1993; Sheridan, 1992) Thus, help with reminiscence provides not only a tool to stimulate interaction, but also a contribution to improved quality of life for the person with dementia and their family. It has been suggested that the 'main impact' of reminiscence is the positive effect it has on communication in general (Woods, 1994).

\section{POSSIBILITIES FOR MULTIMEDIA}

Recent work on using videos to present life histories for people with dementia has shown that new technologies, where sensitively and appropriately applied, can bring a substantial added impact to supportive and therapeutic activities for people with cognitive problems (Cohen 2000). Multi-media systems could provide a richness of interaction which is particularly appropriate for those elderly people with diminishing sensory and intellectual capabilities. For people who are quite severely affected by dementia, with no functioning short-term memory, computer-aided reminiscence could act as an aid to conversation with their families and caregivers.

A reminiscence experience based on a computer, using multimedia techniques, might provide a livelier and more engaging form of reminiscence activity and thus communication support than previously available methods. Reminiscence sessions make use of a variety of separate media. It can be very time consuming searching for a particular photo, sound or film clip. Bringing all these media together into a multi-media system could mean a more integrated framework for a reminiscence session and save valuable time. Multimedia technology affords the seamless inclusion of text, photographs, graphics, sound and film recordings, and also the ability to link the various items together in a dynamic and flexible way. The effective design of multimedia systems is currently in its early stages. If it is not well, done, the potential benefits of multimedia may not be realised. As Preece pointed out: '... the key question is how can this technology be harnessed to facilitate learning and human endeavour ... if such systems are not well designed ... they will create psychological problems for users' (Preece, 1993).

A 'hypermedia' (information link) structure has proved to be very powerful in establishing the popularity of the World Wide Web on the internet. The user is invited to interact with the material presented in a more lively way than by just looking at text and pictures on a page. Interestingly, the highly flexible and 
multidimensional nature of hypermedia, which has been cited as a potential navigation problem for users may in fact be of benefit for people with memory loss, in that it does not put any penalty on 'losing the place' (Conklin, 1987; Alm et al., 1992; McKerlie \& Preece, 1992; Maddy \& Brouwer-Janse, 1996; Peiris, Gregor \& Alm, 2000). Whatever place the user is in is the right place to be. Exploring and 'getting lost' are actively encouraged as strategies to enjoy experiencing the material. The design challenge for a multimedia system which could act as a communication support is to make the interface engaging while at the same time having it encourage its use as a prompt for conversation away from the screen. The idea is for the user to be prompted into talking about something relevant to their own experience, and when they are finished, to help them locate quickly another topic which they would like to talk about. The multimedia display in this way should act as far as possible as a kind of adjunct visual and auditory memory for the person with cognitive difficulties. The display would ideally present material as if 'in the mind's eye'.

Work has been carried out through a number of projects at Dundee University to test the feasibility and effectiveness of such a multi-media reminiscence system and communication support. It will be essential that such a system be easy to operate, by carers and people with dementia. It is also important that the experience offered be one that can be enjoyed without relying on short term memory.

To test different ways of interacting with such a system, and to experiment with ways of engaging the attention of and providing entertainment for people with dementia, a number of computer based games were devised and evaluated by people with dementia and their carers. The games took as their starting point the work of Cohen, who has devised a board game for people with dementia to enjoy with their families and friends. The game has no competition, no finishing point, and does not rely on memory for successful play (Cohen, 2000). We developed two prototype computer based games with similar features. They invited the user to press a button on the touch screen, which then activated an animation sequence and music. This sequence ended in the production of a graphic and some text, which was designed to elicit some comments from the user. In use, these simple computer games demonstrated the effectiveness of touchscreens with people who were not familiar with computers, including people with dementia. The games were seen to be engaging, and were enjoyed by people with dementia without the need for an effective short-term memory.

In order to explore ways of presenting and organising reminiscence material in a multi-media presentation, a project was carried out with a group of older people at a community centre who were interested in learning about computers. The project developed a tool which older people could use to create a personal reminiscence website, based on both publicly available and their own material. These were people for whom dementia was not a problem. The project aimed to discover how acceptable the use of this technology would be for the purpose, with a group of older people. In order to elicit personal material easily, the system used a combination of pre-stored material and material supplied by the user, including news cuttings, recipes, graphics and text.

The material was assembled with the help of a structured dialogue with the user which incorporated computer interviewing techniques, giving an interactive question and answer session that evoked memories whilst allowing users the freedom to answer in their own words. The system then combined the person's own material with the prestored material, and automatically compiled it for them into an attractive and easy to navigate website. It was concluded that presenting a tool specifically designed for older people, and one which they have a good motivation for using, can help older people with few 
computing skills to learn to use the new technology. As well as participating in an enjoyable reminiscence session older people were learning about computers and the World Wide Web. Creating a personal page brought about a sense of achievement in 'mastering' new technology.

This project had a number of aspects. It explored ways to help elderly people to use technology easily, and in a way which they found rewarding and relevant. It was also an investigation of ways to use multimedia to present reminiscence material in an engaging and interesting way. A process of iterative design was used, with continuous feedback from users on a series of prototypes they were shown.

An investigation was then undertaken to determine which aspects of multimedia would be most helpful for such a reminiscence experience specifically for people with dementia, and the best way to present this material. A number of prototype interfaces for a multimedia reminiscence experience were developed. These included text, photographs, videos and songs from the past life of the city. The materials were collected with the assistance of the University and Dundee City archives and library, and the Dundee Heritage Project. The prototypes were demonstrated for people with dementia and staff at a day centre run by Alzheimers Scotland Action on Dementia. The following questions were posed and conclusions drawn from these evaluation sessions :

\section{Is it better for the display to use the metaphor of a real-life scrapbook or just provide very simple screen display?}

Staff members tended to prefer the simulated scrapbook presentation, but interestingly, this was a reversal of the preference shown by the group of people who had dementia, the majority preferring the simpler screen presentation. The preference shown for the simpler presentation could be due to reduced cognitive ability. Having a simulated book presentation may give the person with dementia more information to process than they are comfortable with. They would first have to see the book and recognise it as such before moving on to seeing the picture.

\section{How should the scrapbook material best be organised - by subject or by medium?}

The majority of the staff evaluators preferred the arrangement by subject saying it was more logical, some were unsure, however no-one showed a preference for the arrangement by media. The clients with dementia reflected these findings. Despite preferring the arrangement being by subject the majority of evaluators could see benefits from having access to both arrangements. It was concluded that for basic reminiscence sessions the arrangement by subject is preferable. However, access to the arrangement by media should be an option, to make the software available for use in other ways.

\section{How does each individual medium add to the reminiscence process, and what effects are produced by the various media - sounds, pictures/, videos, music?}

It was found that with videos we used the clients were only able to strongly identify with them when they triggered off specific personal memories, whereas songs and photographs were more generally

appreciated. Most of the videos and photographs and all the songs were able to spur conversations however. Attention stayed longest with the songs, which were particularly enjoyed when played repeatedly with everyone singing along. The staff on the other hand felt that some individual clients 
enjoyed particular videos most. It is clearly important what the subject of the video is, and this can be so variable that at this stage a firm conclusion is not possible.

One general finding was that the multimedia presentation produced a great deal of interest and motivation from the people with dementia. Staff were also very keen to see the idea developed further. What these studies highlighted was the need for a more thorough exploration of ways in which this technology can be most effective as a support for satisfying conversation. A current project is taking a multidisciplinary approach to answering this question by developing a fully functioning reminiscence experience and communication support.

\section{A MULTIDISCIPLINARY PROJECT TO DEVELOP A REMINISCENCE SYSTEM AS A COGNITIVE AND COMMUNICATION AID}

We feel it is important to take a multidisciplinary approach in order to make the multimedia reminiscence system as engaging and effective as possible. From our previous work it is apparent that such a system will need to have sophisticated and reliable software, an engaging and well-designed presentation of the media, and it will need to be based on sound psychological and social principles underlying the interaction. The development of the architecture, navigational methods, and content of the reminiscence experience, along with the collation and digitalisation of an extensive audio/visual archive of material offers a demanding design and development challenge. The researchers are drawn from the fields of computer aided design, applied computing, and the psychology of dementia. The interface and visual look of the system is being devised by a professional graphics designer using a multimedia design package. A software developer with human-computer interaction expertise is carrying out the design and coding of the system structure and navigation methods, designing and building the multimedia database and developing the authoring system. A psychologist is responsible for providing design guidance throughout the development of the system, for creating and maintaining links with potential users and clinical professionals, for giving feedback on the system design as it progresses, and for carrying out the formal evaluations. Design and content advice will also be provided throughout the project by Dundee Social Work Department and Alzheimer Scotland Action on Dementia.

A study has been carried out in day-care settings as to what reminiscence practices are currently employed, what works best and how such sessions might be improved by a multi-media system. The findings suggest that an emphasis should be placed on reminiscence activities that are failure-free and that these should be as relevant as possible to the individual. Contacts with managers and other staff members of both Alzheimer Scotland and Dundee Social Work Department are being maintained and 'link' members of staff have been identified. A group of about 40 people with dementia have been contacted to take part in evaluating the material as it is produced. A further 25 carers and family members are also taking part as sources of ideas for the system and as evaluators of it. A number of sample themes and content items that the system might include have been developed and evaluated by this group.

The identification of appropriate software and hardware has been made : a professional multimedia authoring system accessing a multi-media database and outputting through the largest commercially 
available LCD touch-panel display. A structure for the multimedia database has been devised. Random scripting in the programming will ensure that the process of involvement need not be repetitive - each use of the material will be a different experience if desired, while an index or search facility would allow for more predictable options.

Photographic data, film footage (both archive and contemporary), local folk songs, sounds and music, are being identified and collected. The iterative design process has seen the creation of a prototype of the system, incorporating material from sound, video and photographic archives. This initial presentation package has been demonstrated to representatives of both Alzheimer Scotland and Dundee Social Work Department.

The evaluation of the system will include the following considerations :

(1) Determining what value will be added to a reminiscence experience by providing multi-media capabilities.

(a) Its effect on maintaining the interest, involvement of the person with dementia

(b) Its effect for carers and family on their enjoyment in keeping company with the person with dementia

(c) Its effectiveness in facilitating reminiscence experiences as a group activity

(d) Exploring its use as both as an experience in which the session is guided by a participating carer or family member, and, potentially, as a standalone system to be enjoyed by the person with dementia on their own

The system is, in these trials, being compared with attempting to provide the same experience with a paper scrapbook, audio tapes, and video tapes.

(2) Determining the optimum way to present the experience:

(a) Configurable by carer, family member

(b) Random pathways through the material chosen by the system

(c) Using hypermedia links as opposed to sequential links

(3) A study of the effect of incorporating personal material into the general collection.

Effects to be examined are

(a) Degree of interest shown by the person with dementia

(b) Degree of interest shown by the carer or family member

(c) Quality of the experience in terms of

(i) Amount of personal reminiscences it triggers

(ii) Views of the experience by the person with dementia and the carer or family member.

Evaluation of the success of the system will consider first its impact on people with dementia, by recording and comparing conversational turns, pointing, laughing, and singing, in a typical period before, during and after using the tool. The system's effect on relatives/family carers of people with dementia is being examined in terms of interactions, speech, smiles, nods, and other non-verbal 
measures such as, arm patting and encouraging. Also measures of satisfaction and well being are being administered plus pre- and post study measures of stress/burden. The system's impact on care staff is also being assessed by examining interactions, along with pre- and post measures of attitudes towards clients they have responsibility for and pre- and post measure of job satisfaction and enjoyment. The outcome measures are designed to evaluate the effect on interactions and whether the effect goes beyond the immediate situation. Such evaluation reflects the aims of this project which are to maintain the participation of people with dementia in social activity, to increase positive interactions between carers and visitors and people with dementia, and to provide an enjoyable activity that does not increase stress in visitors, professional care staff or people with dementia.

\section{FINDINGS SO FAR}

The project has reached its first stage, and evaluators have been shown a variety of possible media to include in the system, and also a demonstration of what the multimedia presentation might be. In general, the participants with dementia enjoyed the sessions. Communication was not always related to the subject suggested by the pictures, however the opportunity to interact alone often appeared to have a positive effect. Although some of the memories communicated were unhappy ones, none of the participants became visibly upset and everyone seemed willing to talk about those memories.

The most happy memories and the most conversation seemed to stem from family centred celebrations such as holidays, Christmases and birthdays. Photographs including people seemed to be better at eliciting conversation than just scenes or objects. This may be due to the fact that the people were seen to be acting out what is usually done during the various events, which might have made it easier for participants to elicit memories of their own involvement in these events in the past.

A number of participants did not seem to identify with photographs which portrayed a more affluent lifestyle than they were used to in their earlier days. Photographs which show an older, more modest lifestyle may therefore be suitable replacements for modern-day representations. Participants sometimes described the content of the photographs as opposed to talking about their own memories. This may be because some of the pictures were not relevant to their own past.

Some of the more visually complex photographs were misinterpreted by the participants, which echoes our earlier findings about keeping the presentations as simple as possible while retaining their capacity to engage and stimulate.

This project will we hope produce a number of results. Its explorations of appropriate ways to employ multimedia in guiding users through a reminiscence experience will provide guidelines for future developments in this promising area. More general applications could include reminiscence sessions for older people without dementia, and ease of navigation for all inexperienced users through interactive multi-media systems. 


\section{CONCLUSION}

The realisation of computers as cognitive prostheses and communication supports for people with dementia will depend on good multidisciplinary co-operation, encompassing not only the software structures needed, but good design, and a grounding in the psychological and social realities of the situation that people with dementia find themselves in. We feel a good starting point for communication support is to exploit intact long-term memories through providing prompts and stimulation for reminiscence conversations. The work we have done on this approach so far has demonstrated that computer -based multimedia systems do seem to have the ability to engage the attention and interest of people with dementia. They are usually able to make use of touch screens to control what happens with the interface. What now needs further exploration is just what features of such an experience will work well, and what features are to be avoided, where the aim is to provide a cognitive and communicational prosthesis that supports and stimulates conversation in a way that enriches the interaction between people with dementia and others who want to maintain contact with them.

\section{ACKNOWLEDGEMENTS}

The first stages of this work were partially funded by the British Council in Japan and through a Lloyds TSB Foundation for Scotland / Royal Society of Edinburgh Research Fellowship. The current multimedia reminiscence project is funded by the Engineering and Physical Sciences Research Council, under the EQUAL programme. The advice, assistance and participation of Alzheimers Scotland Action on Dementia and Dundee City Council Social Work Department has been essential to this work.

\section{REFERENCES}

Alm, N., Arnott, J., Newell, A. (1990) Hypertext as a host for an augmentative communication system in Proceedings of the European Conference on the Advancement of Rehabilitation Technology (Maastricht, The Netherlands, 1990), ECART, 14.4a-14.4b

Alm, N., Arnott, J., Newell, A. (1992) Prediction and conversational momentum in an augmentative communication system. Communications of the ACM. Vol 35, No 5. pp 46-57.

Alm, N., Todman, J., Elder, L., Newell, A. (1993) Computer aided conversation for severely physically impaired non-speaking people. Proceedings of INTERCHI'93, pp 236-241.

Alm, N. , Waller, A., Newell, A.F. (1996) Developing computer-based cognitive prostheses. Communication ... Naturally -- Proceedings of the Fourth ISAAC Research Symposium. Västerås, Sweden: Mälardalen University Press. pp 157-165.

Arnott, J.L. (1990) The communication prosthesis : a problem of human-computer integration. Proceedings of European Conference on the Advancement of Rehabilitation Technology (ECART), Maastricht, The Netherlands 5-8 November 1990. Contribution 3.1, (5pp). 
Astell, A., Harley, T. (1998) Naming problems in dementia: semantic or lexical? Aphasiology, Vol 12, pp 357-374.

Azuma, T., Bayles, J. (1997) Memory impairments underlying language difficulties in dementia. Topics in Language Disorders, Vol 18, pp 58-71.

Baines, S., Saxby, P., Ehlert, K. (1987) Reality orientation and reminiscence therapy: a controlled cross over study of elderly confused people. British Journal of Psychiatry, 151, pp 222-231.

Cohen, G. (2000) Two new intergenerational interventions for Alzheimer's disease patients and families. American Journal of Alzheimer's Disease. Vol 15 No 3. pp 137-142.

Cole, E. (1999) Cognitive prosthetics: an overview to a method of treatment. NeuroRehabilitation. Vol 12. pp 39-51.

Conklin, J. (1987) Hypertext: An introduction and a survey. IEEE Computer, pp 17-41.

Dye, R., Alm, N., Arnott, J.L., Harper, G., Morrison, A. (1998) A script-based AAC system for transactional interaction. Natural Language Engineering, Vol 4 No 1, pp 57-71.

Engelbart, D.C. (1963) A conceptual framework for the augmentation of man's intellect. In Vistas in Information Handling. Washington DC: Spartan Books, pp 1-13.

Feil, Naomi (1993) The Validation Breakthrough. Health Professions Press, Maryland.

Finnema, E., Dröes, R-M, Ribbe, M., Van Tilburg, W. (1999) The effects of emotion-oriented approaches in the care for persons suffering from dementia: a review of the literature. International Journal of Geriatric Psychiatry, Vol 15, pp 141-161.

Jackson, A. (1991) To reminisce or not to reminisce. Irish Journal of Psychological Medicine, Vol 8, pp 147-148.

Kirsh, N., Levine, S., Fallon-Krueger, M., Jaros, L. (1987) The microcomputer as an 'orthotic' device for patients with cognitive deficits. Journal of Head Trauma Rehabilitation. Vol 2 No 4, pp 77-86.

Kitwood, T. (1990) The dialectics of dementia: with particular reference to Alzheimer's disease. Ageing and Society, Vol 10, pp 177-196.

Maddy, D. Brouwer-Janse (1996) User centred design, the contour of multimedia, theoretical and empirical developments, Academia Research Monograph 19. Ch12. Luton: John Libbey Media.

McKerlie, D and Preece, J. (1992) The hypermedia effect: more than just the sum of its parts, in Proceedings of the St.Petersburg HCI Conference, pp 115-127.

Peiris, R., Gregor, P., Alm, N. (2000) The effects of simulating human conversational style in a computer-based interview. Interacting with Computers, Vol 12 No 6, pp 635-650. 
Preece, J. (1993) Hypermedia, multimedia and human factors. In R. Phillips, (Ed.) Interactive Multimedia. London: Kogan Page Ltd., London.

Rau, Marie. T. (1993) Coping with Communication Challenges in Alzheimer's Disease. California: Singular Publishing Group Inc., California.

Sheridan, Carmel (1992) Failure-free Activities for the Alzheimer's Patient. London: Macmillan Press, London.

Thompson, P. (1978) The Voice of the Past: Oral History. Oxford University Press, Oxford.

U.S. Bureau of the Census Report 1995.

Vanderheiden, G. (1990) Applications of artificial intelligence to the needs of persons with cognitive impairments: the Companion aid. Proceedings of RESNA International 1992. pp 388-390.

Waller, A., Dennis, F., Cairns, A.Y., Brodie, J.K., Newell, A.F., Morrison, K. (1995) Evaluating the use of TalksBac with non-fluent dysphasic adults. Proceedings of RESNA '95. Vancouver, June 1995. pp 109-111.

Woods, R. T. (1994) Management of memory impairment in older people with dementia. International Review of Psychiatry, Vol 6, pp 153-161.

Woods, R. T (1999) Psychological therapies in dementia. In R. T. Woods (Ed.) Psychological Problems of Ageing. Chichester: Wiley. 